This is the author's versión of a work that was accepted for publication in Electronic notes in discrete mathematics (Elsevier). Changes resulting from the publishing process, such as peer review, editing, corrections, structural formatting, and other quality control mechanisms may not be reflected in this document. Changes may have been made to this work since it was submitted for publication. A definitive versión was subsequently published in Barrolleta, Roland D. and Villanueva, M. "Partial permutation decoding for binary linear Hadamard codes" in Electronic notes in discrete mathematics, vol. 46 (Sep. 2014), p. 35-42. The final versión is available at DOI 10.1016/j.endm.2014.08.006

\title{
Partial permutation decoding for binary linear Hadamard codes *
}

\author{
R. D. Barrolleta ${ }^{1}$ and M. Villanueva ${ }^{2}$ \\ Departament d'Enginyeria de la Informació i de les Comunicacions \\ Universitat Autònoma de Barcelona \\ Cerdanyola del Vallès, Spain
}

\begin{abstract}
Permutation decoding is a technique which involves finding a subset $S$, called PDset, of the permutation automorphism group PAut $(C)$ of a code $C$ in order to assist in decoding. A method to obtain $s$-PD-sets of size $s+1$ for partial permutation decoding for the binary linear Hadamard codes $H_{m}$ of length $2^{m}$, for all $m \geq 4$ and $1<s \leq\left\lfloor\left(2^{m}-m-1\right) /(1+m)\right\rfloor$, is described. Moreover, a recursive construction to obtain $s$-PD-sets of size $s+1$ for $H_{m+1}$ of length $2^{m+1}$, from a given $s$-PD-set of the same size for the Hadamard code of half length $H_{m}$ is also established.
\end{abstract}

Keywords: Permutation decoding, Hadamard codes, automorphism groups.

\section{Introduction}

Let $\mathbb{F}_{2}^{n}$ be the set of all binary vectors of length $n$. The Hamming weight wt $(v)$ of a vector $v \in \mathbb{F}_{2}^{n}$ is the number of nonzero coordinates in $v$. The Hamming

* Research partially supported by the Spanish MICINN under Grants TIN2010-17358 and TIN2013-40524-P, and by the Catalan AGAUR under Grant 2014SGR-691.

1 Email: rolanddavid.barrolleta@uab.cat

2 Email: merce.villanueva@uab.cat 
distance $d(u, v)$ between two vectors $u, v \in \mathbb{F}_{2}^{n}$ is the number of coordinates in which $u$ and $v$ differ, that is, $d(u, v)=\operatorname{wt}(u+v)$. Let $\mathbf{0}$ and $\mathbf{1}$ denote the all-zero and all-one vectors, respectively.

A binary code $C$ of length $n$ is a subset of $\mathbb{F}_{2}^{n}$. The vectors of a code $C$ are called codewords and the minimum (Hamming) distance, denoted by $d$, is the smallest distance between any pair of different codewords in $C$. We said that a code $C$ is a $t$-error-correcting code if it corrects all error vectors of weight at most $t$ and does not correct at least one error vector of weight $t+1$, so $t=\left\lfloor\frac{d-1}{2}\right\rfloor[7]$. A binary code $C$ is linear if it is a $k$-dimensional subspace of $\mathbb{F}_{2}^{n}$. A generator matrix for a linear code $C$ of length $n$ and dimension $k$ is any $k \times n$ matrix $G$ whose rows forms a basis of $C$.

Let $C$ be a binary code of length $n$. For a vector $v \in \mathbb{F}_{2}^{n}$ and a set $I \subseteq$ $\{1, \ldots, n\}$, we denote by $v_{I}$ the restriction of the vector $v$ to the coordinates in $I$ and by $C_{I}$ the set $\left\{v_{I} \mid v \in C\right\}$. For example, if $I=\{1, \ldots, k\}$ and $v=\left(v_{1}, \ldots, v_{n}\right)$, then $v_{I}=\left(v_{1}, \ldots, v_{k}\right)$. Suppose that $C$ has size $|C|=2^{k}$. A set $I \subseteq\{1, \ldots, n\}$ of $k$ coordinate positions is an information set for $C$ if $\left|C_{I}\right|=2^{k}$. For each information set $I \subseteq\{1, \ldots n\}$ of $k$ coordinates positions, the set $\{1, \ldots, n\} \backslash I$ of the remaining $n-k$ coordinate positions is a check set for $C$. If $C$ is linear, we can label the $i^{\text {th }}$ coordinate position by the $i^{\text {th }}$ column of a generator matrix of $C$, so we will consider any information set (or check set) not only as a set of coordinate positions, but also as the set of vectors representing these positions.

Let $\operatorname{Sym}(n)$ be the symmetric group of permutations on the set $\{1, \ldots, n\}$ acting on $\mathbb{F}_{2}^{n}$ by permuting the coordinates of each vector. More specifically, for every vector $v=\left(v_{1}, \ldots, v_{n}\right) \in \mathbb{F}_{2}^{n}$ and permutation $\sigma \in \operatorname{Sym}(n)$, we define $\sigma\left(v_{1}, \ldots, v_{n}\right)=\left(v_{\sigma^{-1}(1)}, \ldots, v_{\sigma^{-1}(n)}\right)$. Then, for any binary code $C$, we denote by PAut $(C)$ the permutation automorphism group of $C$, that is, $\operatorname{PAut}(C)=\{\sigma \in \operatorname{Sym}(n) \mid \sigma(C)=C\}$.

Permutation decoding is a technique, introduced in [7] by MacWilliams, which involves finding a subset $S$, called PD-set, of the permutation automorphism group $\operatorname{PAut}(C)$ of a code $C$ in order to assist in decoding. The method works as follows: Given a $t$-error-correcting linear code $C \subseteq \mathbb{F}_{2}^{n}$ with fixed information set $I$, we denote by $y=x+e$ the received vector, where $x \in C$ and $e$ is the error vector. Suppose that at most $t$ errors occur, that is, $\operatorname{wt}(e) \leq t$. The aim of permutation decoding is to move all errors in a received vector out the information positions, that is, move the nonzero coordinates of $e$ out of $I$, by using an automorphism of the code.

Let $C$ be a $t$-error-correcting linear code with information set $I$. A subset $S \subseteq$ PAut $(C)$ is a $P D$-set for the code $C$ if every $t$-set of coordinate positions 
is moved out of the information set $I$ by at least one element of the set $S$. Equivalently, a subset $S \subseteq$ PAut $(C)$ is an $s$-PD-set if every $s$-set of coordinate positions is moved out of $I$ by at least one element of $S$, where $1 \leq s \leq t$.

Let $S_{m}$ be the binary simplex code of length $2^{m}-1$, dimension $m$ and minimum distance $2^{m-1}$ with generator matrix $G_{S_{m}}$ containing as column vectors the $2^{m}-1$ nonzero vectors from $\mathbb{F}_{2}^{m}$, with the basis elements $e_{i}^{T}, i \in$ $\{1, \ldots, m\}$, in the first $m$ positions. We take the set of standard basis elements of $\mathbb{F}_{2}^{m}$ to be the information set $I_{m}$ of this code, that is, $I_{m}=\left\{e_{1}, \ldots, e_{m}\right\}$. Let $H_{m}$ be the binary linear Hadamard code of length $2^{m}$, that is, the extended code of the simplex code $S_{m}$ with generator matrix $G_{H_{m}}$ constructed from $G_{S_{m}}$ by adding an all-one row vector and an all-zero column vector as follows:

$$
G_{H_{m}}=\left(\begin{array}{cc}
1 & 1 \\
\mathbf{0} & G_{S_{m}}
\end{array}\right) .
$$

Now we consider as information set for $H_{m}$ the set $\mathcal{I}_{m}=\left\{w_{1}, \ldots, w_{m+1}\right\}=$ $\{(1,0, \ldots, 0),(1,1, \ldots, 0), \ldots,(1,0, \ldots, 1)\}$ consisting of the first $m+1$ column vectors from the matrix $G_{H_{m}}$ considered as row vectors. The check set $\mathcal{C}_{m}$ for $H_{m}$ is the set containing the remaining column vectors from the matrix $G_{H_{m}}$ considered as row vectors and denoted by $\mathcal{C}_{m}=\left\{w_{m+2}, \ldots, w_{2^{m}}\right\}$.

It is a well-know fact that $\operatorname{PAut}\left(S_{m}\right)=G L(m, 2)$, where $G L(m, 2)$ is the general linear group of degree $m$ over $\mathbb{F}_{2}$. It is also known that $\operatorname{PAut}\left(H_{m}\right)=$ $A G L(m, 2)[8]$. Recall that the affine group $A G L(m, 2)$ consists of all mappings $\alpha: \mathbb{F}_{2}^{m} \rightarrow \mathbb{F}_{2}^{m}$ of the form $\alpha\left(x^{T}\right)=A x^{T}+b^{T}$ for $x \in \mathbb{F}_{2}^{m}$, where $A \in G L(m, 2)$ and $b \in \mathbb{F}_{2}^{m}$, together with the function composition as the group operation. The monomorphism $\varphi: A G L(m, 2) \rightarrow G L(m+1,2)$,

$$
\varphi(b, A)=\left(\begin{array}{ll}
1 & b \\
0 & A
\end{array}\right)
$$

defines an isomorphism between $A G L(m, 2)$ and the subgroup of $G L(m+1,2)$ consisting of all nonsingular matrices whose first column is $(1,0, \ldots, 0)$. From now on, we identify the $A G L(m, 2)$ with this subgroup.

Now, we describe how to identify a permutation $\sigma \in \operatorname{PAut}\left(H_{m}\right) \subseteq \operatorname{Sym}\left(2^{m}\right)$ with a matrix $B \in A G L(m, 2)$. Recall that each coordinate position can be labelled by the corresponding column of the generator matrix $G_{H_{m}}$ given in (1). The first $m+1$ coordinate positions are labelled by the vectors of the information set $\mathcal{I}_{m}$ and the remaining coordinate positions are represented by the vectors of the check set $\mathcal{C}_{m}$. The vector $w_{i}$ represents the $i^{\text {th }}$ position, for 
all $i \in\left\{1, \ldots, 2^{m}\right\}$. Note that an index $i \in\{1, \ldots, m+1\}$ represents a position in $\mathcal{I}_{m}$ and an index $i \in\left\{m+2, \ldots, 2^{m}\right\}$ a position in $\mathcal{C}_{m}$. Thus, $w_{i} B=w_{j}$ will denote that the $i^{t h}$ position of a codeword moves to the $j^{\text {th }}$ position of that codeword. Therefore, any matrix $B \in A G L(m, 2)$ can be seen as an element of $\operatorname{PAut}\left(H_{m}\right) \subseteq \operatorname{Sym}\left(2^{m}\right)$. Along the paper, we will represent PD-sets for $H_{m}$ as subsets of matrices of the affine group $A G L(m, 2)$.

In [3], it is shown how to find $s$-PD-sets of size $s+1$ that satisfy the Gordon-Schönheim bound for partial permutation decoding for the binary simplex code $S_{m}$, for all $m \geq 4$ and $1<s \leq\left\lfloor\frac{2^{m}-m-1}{m}\right\rfloor$. In this paper, we establish similar results for the binary linear Hadamard code $H_{m}$, for all $m \geq 4$ and $1<s \leq\left\lfloor\frac{2^{m}-m-1}{1+m}\right\rfloor$, following the same techniques as the ones described in [3]. In [9], a 2-PD-set of size 5 and 4-PD-sets of size $\left(\begin{array}{c}m+1 \\ 2\end{array}\right)+2$ are found for binary linear Hadamard codes $H_{m}$, for all $m>4$. As a consequence, 3-PD-sets of size $\left(\begin{array}{c}m+1 \\ 2\end{array}\right)+2$ are also found for these codes. Small PD-sets that satisfy the Gordon-Schönheim bound have been found for binary Golay codes $[4,10]$ and for the binary simplex code $S_{4}[5,6]$.

This work is organized as follows. In Section 2, we adapt the so-called Gordon-Schönheim bound for $H_{m}$ and we define a bound that allow us to obtain $s$-PD-sets of size $s+1$ for $H_{m}$. In Section 3, we provide a criterion on subsets of matrices of $A G L(m, 2)$ to be an $s$-PD-set of size $s+1$. In Section 4, we define a recursive construction to obtain $s$-PD-sets of size $s+1$ for $H_{m+1}$ from a given $s$-PD-set of the same size for $H_{m}$. Finally, in Section 5 , we show the conclusions and a further research on this topic.

\section{Bound on the minimum size of $s$-PD-sets for $H_{m}$}

There is a well-known bound on the minimum size of PD-sets for linear codes based on the length, the dimension and the minimum distance of such codes.

Proposition 2.1 [4] Let $C$ be a t-error correcting linear code of length $n$, dimension $k$ and minimum distance $d$. Let $r=n-k$ be the redundancy of $C$. If $S$ is a $P D$-set for $C$, then

$$
|S| \geq\left\lceil\frac{n}{r}\left\lceil\frac{n-1}{r-1}\left\lceil\ldots\left\lceil\frac{n-t+1}{r-t+1}\right\rceil \ldots\right\rceil\right\rceil\right\rceil .
$$

The above inequality is often called the Gordon-Schönheim bound. Recall that a linear code with minimum distance $d$ can correct up to $\left\lfloor\frac{d-1}{2}\right\rfloor$ errors, so for the binary linear Hadamard code $H_{m}$, we have that its error-correcting 
capability, denoted by $t_{m}$, is $t_{m}=2^{m-2}-1$. We do not take into account the case $m=3$ in our results since $t_{3}=1$. The Gordon-Schönheim bound can be adapted to $s$-PD-sets for all $s$ up to the error correcting capability of the code. We compute the function $g_{m}(s)$ defined by the right side of this bound given in Proposition 2.1 in the particular case of the binary linear Hadamard code $H_{m}$, for all $1 \leq s \leq t_{m}$. The minimum value of $g_{m}(s)$ is also computed.

Lemma 2.2 Let $m$ be an integer, $m \geq 4$. Let $H_{m}$ be the binary linear Hadamard code. For $1 \leq s \leq t_{m}$,

$$
g_{m}(s)=\left\lceil\frac{2^{m}}{2^{m}-m-1}\left\lceil\frac{2^{m}-1}{2^{m}-m-2}\left\lceil\ldots\left\lceil\frac{2^{m}-s+1}{2^{m}-m-s}\right\rceil\right\rceil \ldots\right\rceil\right\rceil \geq s+1,
$$

where $t_{m}=2^{m-2}-1$ is the error-correcting capability of $H_{m}$.

The smaller the size of the PD-set is, the more efficient permutation decoding becomes. Because of this, we will focus on the case when we have that $g_{m}(s)=s+1$. Let $m$ be an integer, $m \geq 4$. For the binary linear Hadamard code $H_{m}$, we define $f_{H_{m}}=\max \left\{s \mid 2 \leq s, g_{m}(s)=s+1\right\}$. For each $H_{m}$, the integer $f_{H_{m}}$ represents the greater $s$ in which we can find $s$-PD-sets of size $s+1$. The following result characterize this parameter from the value of $m$.

Lemma 2.3 For $m \geq 4, f_{H_{m}}=\left\lfloor\frac{2^{m}-m-1}{1+m}\right\rfloor$.

\section{Finding $s$-PD-sets of size $s+1$ for $H_{m}$}

Let $M$ be a matrix of $G L(m, 2)$. We can regard the rows of $M$ as row vectors and consider the set $V=\left\{v_{1}, \ldots, v_{m}\right\}$ consisting of such row vectors. We define $M^{*}$ as the matrix with rows given by $V^{*}=\left\{v_{1}, v_{1}+v_{2}, \ldots, v_{1}+v_{m}\right\}$. We denote by $I d_{m}$ the $m \times m$ identity matrix.

An $s$-PD-set of size $s+1$ meets the Gordon-Schönheim bound for correction of $s$ errors if $s \leq f_{H_{m}}$. The following proposition provides us a condition on sets of matrices of $A G L(m, 2)$ in order to be $s$-PD-sets of size $s+1$.

Proposition 3.1 Let $H_{m}$ be the binary linear Hadamard code of length $n=$ $2^{m}$, with $m \geq 4$. Let $P_{s}=\left\{M_{i} \mid 0 \leq i \leq s\right\}$ be a set of $s+1$ matrices in $A G L(m, 2)$. Then, $P_{s}$ is an $s-P D$-set of size $s+1$ for $H_{m}$ if and only if no two matrices $\left(M_{i}^{-1}\right)^{*}$ and $\left(M_{j}^{-1}\right)^{*}$ for $i \neq j$ have a row in common. Moreover, any subset $P_{k} \subseteq P_{s}$ of size $k+1$ is a $k$-PD-set for $k \in\{1, \ldots, s\}$. 
Example 3.2 The set of matrices $P_{2}=\left\{I d_{5}, M_{1}, M_{2}\right\}$, where

$$
M_{1}=\left(\begin{array}{lllll}
1 & 1 & 1 & 1 & 1 \\
0 & 0 & 0 & 0 & 1 \\
0 & 0 & 1 & 0 & 0 \\
0 & 1 & 0 & 0 & 1 \\
0 & 0 & 1 & 1 & 0
\end{array}\right) \quad \text { and } \quad M_{2}=\left(\begin{array}{lllll}
1 & 1 & 1 & 1 & 1 \\
0 & 1 & 0 & 0 & 1 \\
0 & 0 & 1 & 1 & 0 \\
0 & 0 & 1 & 0 & 0 \\
0 & 0 & 0 & 0 & 1
\end{array}\right)
$$

is a 2-PD-set for the binary linear Hadamard code $H_{4}$ of length 16 . Note that $P_{2} \subset A G L(4,2) \subset G L(5,2)$. It is straightforward to check that $I d_{5}^{*}$,

$$
\left(M_{1}^{-1}\right)^{*}=\left(\begin{array}{ccccc}
1 & 0 & 0 & 1 & 1 \\
1 & 1 & 0 & 0 & 1 \\
1 & 0 & 1 & 1 & 1 \\
1 & 0 & 1 & 1 & 0 \\
1 & 1 & 0 & 1 & 1
\end{array}\right) \quad \text { and } \quad\left(M_{2}^{-1}\right)^{*}=\left(\begin{array}{lllll}
1 & 1 & 1 & 0 & 0 \\
1 & 0 & 1 & 0 & 1 \\
1 & 1 & 1 & 1 & 0 \\
1 & 1 & 0 & 1 & 0 \\
1 & 1 & 1 & 0 & 1
\end{array}\right)
$$

have no rows in common. In addition, note that $f_{H_{4}}=2$, so no $s$-PD-set of size $s+1$ can be found for $s \geq 3$. We can also observe that $f_{H_{4}}=2<$ $3=t_{4}$, where $t_{4}$ is the error-correcting capability of $H_{4}$. In fact, the value of the bound $f_{H_{m}}$ is always smaller than $t_{m}$, for all $m \geq 4$. Finally, $P_{2}$ can be regarded as a subset of $\operatorname{Sym}(16)$. In this case, we obtain the 2-PDset $\left\{i d, \sigma_{1}, \sigma_{2}\right\}$ where $\sigma_{1}=(1,14,11,9,6,10,13,3,15,5,16,2,12,8)(4,7)$ and $\sigma_{2}=(1,14,11,2,7,9,5,12,3,16,13,6)(4,15,8,10)$.

Let $S$ be an $s$-PD-set of size $s+1$. The set $S$ is a nested $s$-PD-set if there is an ordering of the elements of $S, S=\left\{\sigma_{0}, \ldots, \sigma_{s}\right\}$, such that $S_{i}=$ $\left\{\sigma_{0}, \ldots, \sigma_{i}\right\} \subseteq S$ is an $i$-PD-set of size $i+1$, for all $i \in\{0, \ldots, s\}$. Note that $S_{i} \subset S_{j}$ if $0 \leq i<j \leq s$ and $S_{s}=S$. From Proposition 3.1, we have two important consequences. The first one is related to how to obtain nested $s$-PD-sets and the second one provides another proof of Lemma 2.3.

Corollary 3.3 Let $m$ be an integer, $m \geq 4$. If $P_{s}$ is an $s$-PD-set of size $s+1$ for the binary linear Hadamard code $H_{m}$, then any ordering of the elements of $P_{s}$ gives nested $k$-PD-sets for $k \in\{1, \ldots, s\}$. 
Corollary 3.4 Let $m$ be an integer, $m \geq 4$. Let $P_{s}$ be an $s$-PD-set of size $s+1$ for the binary linear Hadamard code $H_{m}$. Then, $s \leq\left\lfloor\frac{2^{m}-m-1}{1+m}\right\rfloor$.

\section{Recursive construction of $s$-PD-sets of size $s+1$}

Given an $s$-PD-set of size $s+1$ for the binary linear Hadamard code $H_{m}$ of length $2^{m}$, where $0 \leq s \leq f_{H_{m}}$, we can construct recursively an $s$-PD-set of the same size for $H_{m^{\prime}}$ of length $2^{m^{\prime}}$, for all $m^{\prime}>m$.

Let $M \in A G L(m, 2)$ and $v=\left(0, v_{2} \ldots, v_{m+1}\right)$ be the last row of the matrix $M$. We define the matrix $M(v) \in A G L(m+1,2)$ as

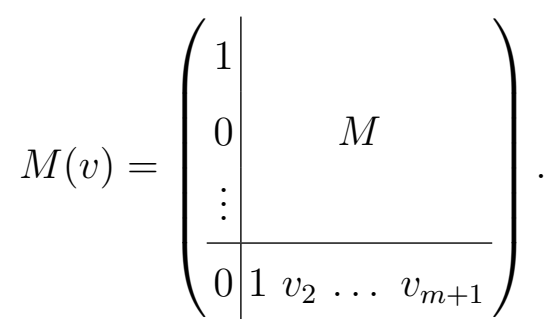

Since the first column of $M(v)$ is $e_{1}=(1,0, \ldots, 0)$, we can guarantee that the first column of $M(v)^{-1}$ is $e_{1}$ as well. Thus, $M(v), M(v)^{-1} \in A G L(m+1,2)$. Also note that $v \in \mathbb{F}_{2}^{m+1}$ depends on each $M \in A G L(m, 2)$.

Proposition 4.1 Let $m$ be an integer, $m \geq 4$, and $P_{s}=\left\{I d_{m+1}, M_{1}, \ldots, M_{s}\right\}$ be an $s-P D$-set of size $s+1$ for the binary linear Hadamard code $H_{m}$. Let $N_{i}=$ $M_{i}^{-1}$, for all $i \in\{1, \ldots, s\}$. Then, $Q_{s}=\left\{I d_{m+2},\left(N_{1}(v)\right)^{-1}, \ldots,\left(N_{s}(v)\right)^{-1}\right\}$ is an $s$-PD-set of size $s+1$ for the binary linear Hadamard code $H_{m+1}$.

Example 4.2 Considering the matrices from the 2-PD-set $P_{2}=\left\{I d_{5}, M_{1}, M_{2}\right\}$ for $H_{4}$ of length 16, given in Example 3.2, matrices $N_{1}(v)=\left(M_{1}^{-1}\right)(v)$ and $N_{2}(v)=\left(M_{2}^{-1}\right)(v)$ are

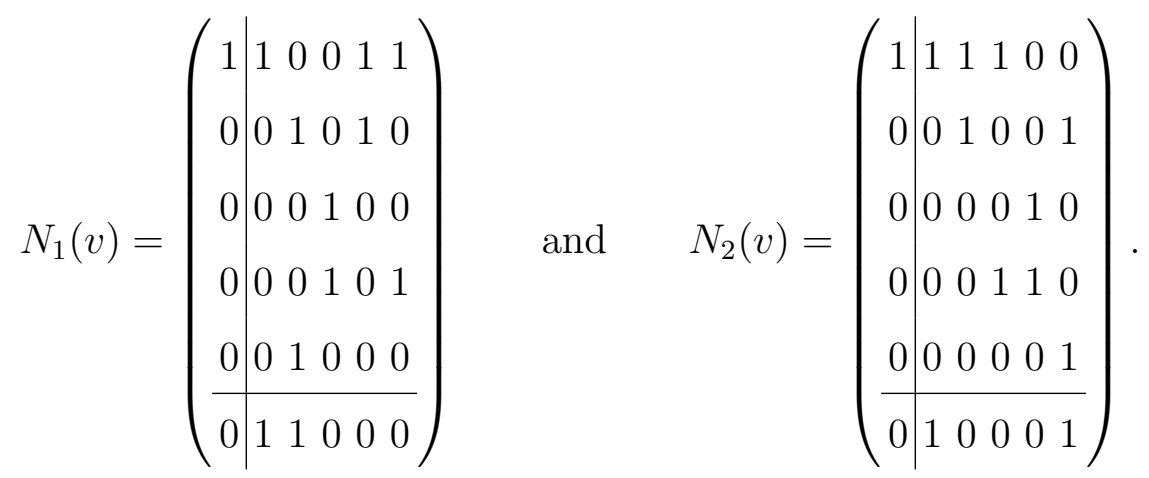


Note that the last row of $N_{1}$ is $v=(0,1,0,0,0)$, and the last row of $N_{2}$ is $v=(0,0,0,0,1)$. Since matrices $I d_{6}^{*},\left(N_{1}(v)\right)^{*}$ and $\left(N_{1}(v)\right)^{*}$ have no rows in common, the set $\left\{I d_{m+2},\left(N_{1}(v)\right)^{-1},\left(N_{2}(v)\right)^{-1}\right\}$ is a 2-PD-set for $H_{5}$.

Note 1 Proposition 4.1 is also true if we define the matrix $M(v)$ taking as vector $v$ any of the last $m$ rows of $M$ instead of the last one as in (2).

Note 2 The bound $f_{H_{m+1}}$ for $H_{m+1}$ cannot be achieve recursively from an $s$ $P D$-set for $H_{m}$. The recursive construction only works when fixing the number $s$ of errors we want to correct and increasing the length of the Hadamard code.

\section{Conclusions and further research}

In this work, we studied how to find $s$-PD-sets for partial permutation decoding for binary linear Hadamard codes. An alternative permutation decoding algorithm for $\mathbb{Z}_{2} \mathbb{Z}_{4}$-linear codes [2] is described in [1]. In particular, it can be applied to Hadamard $\mathbb{Z}_{2} \mathbb{Z}_{4}$-linear codes. Nevertheless, this method assumes that we know an appropriate PD-set for such codes. Further work will be study how to find $s$-PD-sets for Hadamard $\mathbb{Z}_{2} \mathbb{Z}_{4}$-linear codes (not necessarily binary linear Hadamard codes) and establish the size of these $s$-PD-sets.

\section{References}

[1] Bernal, J. J., J. Borges, C. Fernández-Córboda, and M. Villanueva, Permutation decoding of $\mathbb{Z}_{2} \mathbb{Z}_{4}$-linear codes, Des. Codes Cryptogr. (2014), DOI 10.1007/s10623-014-9946-4.

[2] Borges, J., C. Fernández-Córdoba, J. Pujol, J. Rifà, and M. Villanueva, $\mathbb{Z}_{2} \mathbb{Z}_{4^{-}}$ linear codes: generator matrices and duality, Des. Codes and Cryptogr. 54 (2010), 167-179.

[3] Fish, W., J. D. Key, and E. Mwambene, Partial permutation decoding for simplex codes, Advances in Mathematics of Comunications 6 (2012), 505-516.

[4] Gordon, D. M., Minimal permutation sets for decoding the binary Golay codes, IEEE Trans. Inform. Theory 28 (1982), 541-543.

[5] Kroll, H.-J., and R. Vicenti, PD-sets related to the codes of some classical varieties, Discrete Math. 301 (2005), 89-105.

[6] Kroll, H.-J., and R. Vicenti, PD-sets for binary RM-codes and the codes related to the Klein quadric and to the Schubert variety of $P G(5,2)$, Discrete Math. 308 (2008), 408-414. 
[7] MacWilliams, F. J., Permutation decoding of systematics codes, Bell System Tech. J. 43 (1964), 485-505.

[8] MacWilliams F. J., and N. J. A. Sloane, "The Theory of Error-Correcting Codes," North-Holland Publishing Company, Amsterdam, 1977.

[9] Seneviratne, P., Partial permutation decoding for the first-order Reed-Muller codes, Discrete Math. 309 (2009), 1967-1970.

[10] Wolfmann, J., A permutation decoding of the (24, 12, 9) Golay code, IEEE Trans. Inform. Theory 29 (1983), 748-750. 